\title{
Nonlinear acoustoelectric transport in a two-dimensional electron system
}

\author{
A. O. Govorov* and A. V. Kalameitsev \\ Institute of Semiconductor Physics, Russian Academy of Sciences, Siberian Branch, 630090 Novosibirsk, Russia \\ M. Rotter, A. Wixforth, and J. P. Kotthaus \\ Sektion Physik der Ludwig-Maximilians-Universität and Center for NanoScience, Geschwister-Scholl-Platz, 1, \\ D-80539 München, Germany \\ K.-H. Hoffmann and N. Botkin \\ Center of Advanced European Studies and Research, Friedensplatz 16, D-53111 Bonn, Germany
}

(Received 30 August 1999; revised manuscript received 22 February 2000)

\begin{abstract}
We study both theoretically and experimentally the nonlinear interaction between an intense surface acoustic wave and a two-dimensional electron plasma in semiconductor-piezocrystal hybrid structures. The experiments on hybrid systems exhibit strongly nonlinear acoustoelectric effects. The plasma turns into moving electron stripes, the acoustoelectric current reaches its maximum, and the sound absorption strongly decreases. To describe the nonlinear phenomena, we develop a coupled-amplitude method for a two-dimensional system in the strongly nonlinear regime of interaction. At low electron densities the absorption coefficient decreases with increasing sound intensity, whereas at high electron density the absorption coefficient is not a monotonous function of the sound intensity. High-harmonic generation coefficients as a function of the sound intensity have a nontrivial behavior. Theory and experiment are found to be in a good agreement.
\end{abstract}

\section{INTRODUCTION}

The interaction between surface acoustic waves (SAW's) and mobile carriers in quantum wells is an important method to study the dynamic properties of two-dimensional (2D) systems. The SAW can trap carriers and induce acoustic charge transport as has been investigated in a number of systems in view of possible device applications. ${ }^{1}$ Also, the SAW-method was applied to study the quantum Hall effects, ${ }^{2-4}$ electron transport through a quantum-point contact, ${ }^{5}$ lateral nanostructures, ${ }^{6}$ and commensurability effects in a $2 \mathrm{D}$ system. ${ }^{7}$ However, all those experiments have been done in the regime of small signals and linear interaction. A recent paper by Rotter et al. ${ }^{8}$ reports strongly nonlinear acoustoelectric effects in a 2D electron gas (2DEG), which become possible in hybrid structures based on $A_{3} B_{5}$ semiconductors and $\mathrm{LiNbO}_{3} \cdot{ }^{9-11}$ In these experiments an intense SAW breaks a 2DEG into moving electron stripes and all characteristics of the acoustoelectric interaction are strongly modified as compared to the linear case. In modern hybrid structures ${ }^{11}$ the SAW-induced potential amplitude can become comparable with the band-gap of a semiconductor. The previous paper ${ }^{8}$ on nonlinear effects in the hybrid structures with a 2 DEG includes a brief qualitative analysis. Here we present a detailed theoretical study of nonlinear acoustoelectric effects in a 2D electron system and develop a coupled-amplitude method for intense SAW's interacting with a 2DEG. Using our theoretical results we can explain main experimental observations. Including the effect of electron diffusion we find a good quantitative agreement between theory and experiment for the case of the SAW absorption coefficient in the nonlinear regime.

Nonlinear acoustic waves in bulk piezocrystals with free carriers were discussed in a number of theoretical papers. ${ }^{12-16}$ The application of a dc voltage to the crystal can result in the current amplification of sound ${ }^{12,14,17}$ and in the formation of stationary nonlinear waves. ${ }^{12,14,16}$ Analytic results in the theory of nonlinear acoustic waves in bulk piezocrystals with free carriers were mostly obtained in the limit of small amplitudes or for the case of very intense acoustic waves. ${ }^{12,13,16}$ For nonlinear SAW's in crystals with a 3D electron gas, a theory was developed in the limit of very high amplitudes, when the SAW bunches electrons near the crystal surface. ${ }^{18}$ Another theoretical aspect related to the generation of the second harmonic of a SAW was studied in Ref. 19 by using the coupled-amplitude method and perturbation theory. A theory of acoustoelectric interactions in a 2D electron system was developed mostly for the linear regime of interaction. ${ }^{20,21}$

Here, we study both theoretically and experimentally the transition from the linear regime of the acoustoelectric interaction to the limit of strongly nonlinear effects in a 2DEG. Our theoretical results are applied for a description of experimental data on hybrid structures. ${ }^{8,11}$ We pay attention to density dependences of the absorption coefficient and the SAWvelocity shift because the electron density is a tunable parameter in experiments on 2D systems. Such dependences were not discussed in detail in the context of $3 \mathrm{D}$ systems. ${ }^{12-16}$ It turns out that for low densities, the absorption coefficient is a decreasing function of the sound intensity caused by the trapping of electrons in the SAW piezoelectric potential. At sufficiently high electron density, however, the absorption coefficient is a nonmonotonous function of the sound intensity. This behavior can be understood in terms of a dynamical screening effect. Also, our quantitative analysis shows that the absorption coefficient at room temperature is strongly reduced due to electron diffusion. A nonmonotonous behavior with increasing sound in- 
tensity was also found for the intensities of higher harmonics in a short device.

The coupled-amplitude method with the introduction of fast and slow variables was used before for a description of bulk systems. ${ }^{14,15}$ However, the formulas from the bulk theory cannot be directly applied to surface waves because of the complex character of the lattice vibrations in a SAW. To develop the coupled-amplitude method in a $2 \mathrm{D}$ system, we introduce a local velocity shift and a local absorption coefficient in an integral form through an electron current, a SAW potential, and an electromechanical coupling coefficient of a microstructure. The resulting formulas can then be applied to any type of SAW's interacting with a 2DEG. Moreover, using our approach, we can find solutions for higher harmonics for arbitrary SAW intensity. This is in contrast with perturbation methods developed earlier for 3D systems and valid at small SAW intensities. ${ }^{19}$ At very high intensities, we find analytical asymptotic dependences for the high-harmonic intensities in a $2 \mathrm{D}$ system.

As has been shown before, the linear approximation holds when $\delta n \ll N_{s}$, where $N_{s}$ is the equilibrium 2DEG density and $\delta n$ is a density perturbation due to a SAW. In the linear theory, the absorption coefficient $\Gamma^{0}$ and the SAW-velocity shift due to the 2DEG $\delta v_{s}^{0}$ are given by the well-known relation ${ }^{20,21}$

$$
-\frac{\delta v_{s}^{0}}{v_{s}^{0}}+i \frac{\Gamma^{0}}{2 q}=\frac{K_{e f f}^{2}(q)}{2} \frac{i \sigma_{0} / \sigma_{m}}{1+i\left(\sigma_{0} / \sigma_{m}+D_{e} q / v_{s}^{0}\right)},
$$

where $q$ is the SAW wave vector, $v_{s}^{0}$ is the sound velocity in the absence of a 2DEG, $\sigma_{0}$ and $D_{e}$ are the 2DEG conductivity and the diffusion coefficient, respectively. $K_{\text {eff }}^{2}$ is the effective electromechanical coupling coefficient. $\sigma_{m}(q)$ $=v_{s}^{0} \epsilon_{\mathrm{eff}}(q) /(2 \pi)$, where $\epsilon_{\mathrm{eff}}(q)$ is the effective dielectric constant in a $2 \mathrm{D}$ system. In most piezoelectric crystals, the coupling $K_{\text {eff }}^{2} \ll 1$. In GaAs, $K_{\text {eff }}^{2}=0.00064$, whereas in the hybrid structures as studied here, it is two orders of magnitude larger, in the range of $0.01-0.05,{ }^{10}$ but still much less than unity. The goal of this paper is to describe the acoustoelectric interaction for the case of large amplitude SAW's, when $\delta n \sim N_{s}$ and the perturbation theory is no longer valid. At the same time, the coupling $K_{\text {eff }}^{2}$ will be assumed to be much less than unity. Below, we will generalize the results following from Eq. (1) for the strongly nonlinear case, when $\delta n \sim N_{s}$.

The paper is organized in the following way. In the first section, we will give the general equations for SAW's on a piezoelectric crystal. The second section is devoted to a coupled-amplitude method developed for the case of intense SAW's. The third and fourth sections are about phenomena related to large-amplitude SAW's in a 2DEG. Then, we will discuss experimental data on the hybrid structures and apply our theoretical results for the interpretation of experiments.

\section{MODEL AND GENERAL EQUATIONS}

In usual GaAs-based microstructures it is very difficult to realize SAW's with high-amplitude potentials because the electromechanical coupling in GaAs is relatively weak. A strong piezoelectric interaction can be achieved in hybrid structures $^{8,9}$ (Fig. 1). Those consist of a semiconductor layer

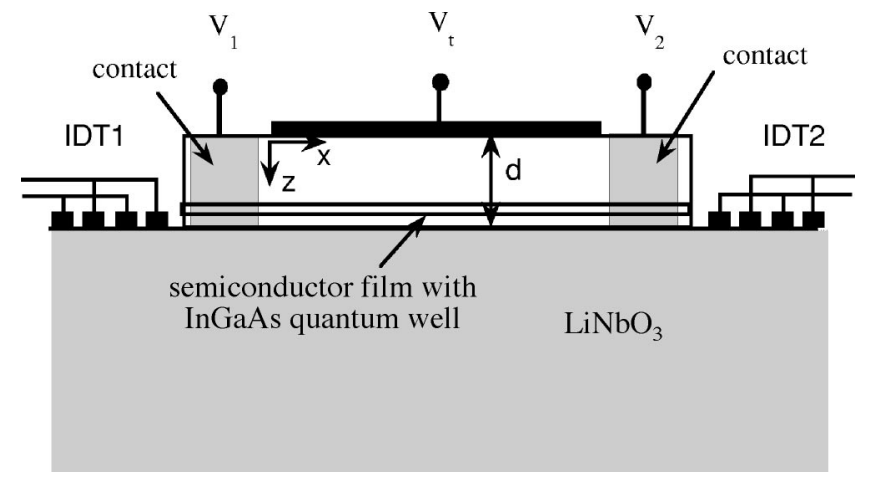

FIG. 1. The cross section of a hybrid semiconductorpiezocrystal structure. An epitaxial lift-off film has a thickness $0.5 \mu \mathrm{m}$. The Ohmic contacts are formed to a $2 \mathrm{D}$ electron gas. The transport gate with applied voltage $V_{t}$ is used to control the conductivity of the electron plasma. A high-frequency (rf) signal is applied to the metal interdigital transducer IDT1 in order to generate surface acoustic waves. A surface acoustic wave propagates though a sample and is detected by the transducer IDT2.

being bonded to a piezoelectric host crystal, in our case, $\mathrm{LiNbO}_{3} .{ }^{8,9}$ The semiconductor layer contains an InGaAsAlGaAs quantum well (QW) with a high-quality 2DEG, to which Ohmic contacts are formed. The distance between the QW and the piezocrystal is only $32 \mathrm{~nm}$, whereas the distance between the QW and the top transport gate is $d=450 \mathrm{~nm}$. In our model $x$ and $y$ are the in-plane coordinates and $z$ is the normal one. The QW plane corresponds to $z=d$, and the SAW travels in the $x$ direction (see Fig. 1). By changing the transport-gate voltage $V_{t}$ one can tune the electron density $N_{s}\left(V_{t}\right)$ in a QW. In this structure, traveling SAW's can induce very strong piezoelectric fields in the semiconductor layer due to the strong piezoelectricity of the host $\mathrm{LiNbO}_{3}$ crystal. A SAW is induced and detected by the metallic interdigital transducers IDT1 and IDT2, respectively, at room temperature. ${ }^{8}$ The acoustoelectric current is measured between two Ohmic contacts labeled 1 and 2 in Fig. 1.

SAW's in a piezoelectric crystal with a $2 \mathrm{D}$ plasma are described by the system of equations ${ }^{22}$

$$
\begin{gathered}
\rho \ddot{u}_{i}=c_{i k l m} \partial_{m} \partial_{k} u_{l}+p_{l i k} \partial_{l} \partial_{k} \phi, \\
\epsilon\left(x_{3}\right) \partial_{i} \partial_{i} \phi-4 \pi p_{i k l} \partial_{i} \partial_{l} u_{k}=-4 \pi e n \delta\left(x_{3}-d\right),
\end{gathered}
$$

where $e=-|e|$ is the electron charge, $\rho$ is the mass density, and $\epsilon$ is the dielectric constant. Further, $c_{i k l m}$ is the elastic tensor, $p_{l i k}$ is the piezoelectric tensor, $u_{k}(x, z, t)$ is the lattice displacement, and $\phi(x, z, t)$ and $n(x, t)$ are the electrostatic potential and the 2D electron density, respectively. In Eqs. (2), (3), we have used the notations $\partial_{l} f=\partial f / \partial x_{l}$ and $\dot{f}$ $=\partial f / \partial t$, and the sum convention for repeated indexes. $t$ is the time, and $x_{3}=z, x_{2}=y$, and $x_{1}=x$ are the coordinates.

In our geometry, the SAW propagating in the $x$ direction is a purely Rayleigh wave, in which only two components of the displacement, $u_{x}$ and $u_{z}$, are nonzero. Hence, the electric field $\mathbf{E}$ is also polarized in the $(x z)$ plane. This case corresponds to the hybrid structures studied in experiments, ${ }^{8-10}$ where the $128^{\circ}$-rotated $Y$ cut of $\mathrm{LiNbO}_{3}$ is used. The surface of the thin GaAs film is (001). The SAW propagates in the [110] direction of GaAs and $X$ direction of $\mathrm{LiNbO}_{3}$. 
The electron 2D plasma is described by the usual hydrodynamic equations

$$
\begin{gathered}
e \dot{n}(x, t)+\frac{\partial j(x, t)}{\partial x}=0, \\
j=-\sigma \frac{\partial \phi(x, d, t)}{\partial x}-e D_{e} \frac{\partial n(x, t)}{\partial x},
\end{gathered}
$$

where $j$ is the 2D electron current, $\sigma=|e| \mu n_{s}(x, t)$ is the 2DEG conductivity, $\mu$ is the mobility, and $D_{e}$ is the electron diffusion coefficient. Equation (5) is valid in the long wavelength limit, when $q l_{e} \ll 1$, where $l_{e}$ is the electron mean free path.

The wave equations (2), (3) should be solved together with the boundary conditions at the surface $z=0$ and at the interface $z=d$. At $z=0$, these conditions are the following: $\phi(z=0)=0 \quad$ and $\quad \sigma_{z i}=c_{z i l m}\left(\partial_{m} u_{l}+\partial_{l} u_{m}\right) / 2+p_{l z k} \partial_{l} \phi=0$. Here $\sigma_{z i}$ is the $z$ component of the stress. The top metal gate is thin and does not influence the boundary condition for the stress tensor. At the semiconductor-piezocrystal interface, $\phi$, $u_{i}$, and $\sigma_{z i}$ should be continuous, and $D_{z}(z=d+\delta)-D_{z}(z$ $=d-\delta)=4 \pi e n$, where $\delta \rightarrow 0$ and $D_{i}=-\epsilon(z) \partial_{i} \phi$ $+2 \pi p_{i k l}\left(\partial_{k} u_{l}+\partial_{l} u_{k}\right)$ is the electric displacement. For simplicity, we assumed above that the 2DEG is located directly at the semiconductor-piezocrystal interface.

\section{COUPLED-AMPLITUDE METHOD}

The system of nonlinear equations (2)-(5) can be simplified in the limit of weak electro-mechanical coupling $K_{\text {eff }}^{2}$ $\sim p^{2} /(c \epsilon) \ll 1$. In this limit, we can introduce two coordinates, the "slow" variable $x$ and the "fast" variable $x_{1}=x$ $-v_{s}^{0} t \cdot{ }^{14,15}$ The solution is of the form (see Appendices A and B),

$$
\begin{aligned}
\mathbf{u}(x, z, t)=\mathbf{u}\left(x, x_{1}, z\right)= & \mathbf{u}_{0}(x, z)+\sum_{n=1,2, \ldots} a_{n}(x) \\
& \times \mathbf{U}^{0}\left[z ; q_{n}+\delta q_{n}(x)\right] e^{i q_{n} x_{1}}+\text { c.c. },
\end{aligned}
$$

$$
\begin{aligned}
\phi(x, z, t)=\phi\left(x, x_{1}, z\right)= & \phi_{0}(x, z)+\sum_{n=1,2, \ldots} a_{n}(x) \\
& \times \Phi^{0}\left[z ; q_{n}+\delta q_{n}(x)\right] e^{i q_{n} x_{1}}+\text { c.c. },
\end{aligned}
$$

This solution is written as a sum of harmonics with wave vectors $q_{n}=n q$, where $q>0$ is the wave vector of the initially generated SAW near $x=0$. The vector $\mathbf{A}^{0}[z ; q]$ $=\left(\mathbf{U}^{0}[z ; q] ; \Phi^{0}[z ; q]\right)$ and the quantity $\delta q_{n}$ are determined by a linear system of equations as given in Appendix B. The envelope functions $a_{n}(x)$ are slowly changing on the scale of $\lambda=2 \pi / q$. It is assumed that the SAW intensity related to the vector $\mathbf{A}^{0}[z ; q]$ is unity and thus the total SAW intensity is $I_{\mathrm{SAW}}=\sum_{n=1,2, \ldots}\left|a_{n}(x)\right|^{2}$. The functions $\mathbf{u}_{0}(x, z)$ and $\phi_{0}(x, z)$ describe the static spatial distributions, that can be induced by a SAW.

The functions $j\left(x, x_{1}, t\right)$ and $n\left(x, x_{1}, t\right)$ can be written in the standard form $f\left(x, x_{1}, t\right)=f_{0}(x)+\Sigma_{n=1,2, \ldots} f_{n}(x) e^{i q_{n} x_{1}}$
+ c.c., where $f_{n}(x)$ is an envelope function. Also, the electric field $\mathbf{E}$ can be written in the way similar to Eq. (7).

The solutions (6), (7) are a sum of linearlike SAW's, which slowly vary in the amplitude and in the $z$ profile. The $z$ distribution of lattice displacement $\mathbf{U}^{0}$ is a sum of exponential functions $\exp \left\{-\gamma_{j}\left[q_{n}+\delta q_{n}(x)\right] z\right\}$, where $\gamma_{j}[q]$ are the coefficients depending also on material constants. ${ }^{22}$ On short distances $(\sim \lambda)$ the envelope functions $a_{n}(x)$ can be regarded as constants and we can solve Eqs. (2)-(5) considering only the "fast" variable $x_{1}, n_{n}(x)$ and $\phi_{n}(x)$ should be considered here as the functions of the parameters $a_{1}, a_{2}, \ldots, n_{0}$, and $E_{0}$. Then, having $n_{n}$ and $\phi_{n}$ as functions of $a_{1}, a_{2}, \ldots, n_{0}$, and $E_{0}$, we can find the behavior of $a_{n}(x)$ on long-range scale $x \sim 1 / \Gamma^{0} \gg \lambda$.

The electrostatic potential is written in a self-consistent way:

$$
\phi\left(x, x_{1}, z\right)=\phi^{\text {ind }}+\phi^{\mathrm{SAW}},
$$

where $\phi^{\text {ind }}$ and $\phi^{\text {SAW }}$ are the potentials induced by a 2DEG and by piezoelectric charges of a SAW. Using Eq. (3) we write

$$
\begin{gathered}
\epsilon(z) \partial_{i} \partial_{i} \phi^{\mathrm{ind}}=-4 \pi e n \delta(z-d), \\
\epsilon(z) \partial_{i} \partial_{i} \phi^{\mathrm{SAW}}=4 \pi p_{i k l} \partial_{i} \partial_{l} u_{k} .
\end{gathered}
$$

$\phi^{\text {ind }}$ and $\phi^{\mathrm{SAW}}$ can be expressed by the harmonic amplitudes $\phi_{n}^{\text {ind }} \quad$ and $\quad \phi_{n}^{\mathrm{SAW}}$. For example, $\phi^{\text {ind }}=\phi_{0}^{\text {ind }}(x, z)$ $+\sum_{n=1,2, \ldots} \phi_{n}^{\text {ind }}(x, z) e^{i q_{n} x_{1}}+$ c.c. In the limit $\Gamma_{\max } \lambda \sim K_{\text {eff }}^{2}$ $\ll 1$, we find from Poisson's equation (see Appendix A) and from the conservation of charge

$$
\phi_{n}^{\text {ind }}(x, d)=\frac{2 \pi e n_{n}(x)}{\epsilon_{e f f}\left(q_{n}\right) q_{n}}, \quad E_{n x}^{\text {ind }}(x, d)=-i q_{n} \phi_{n}^{\text {ind }}(x, d),
$$

$$
j_{n}(x)=v_{s}^{0} e n_{n}(x),
$$

where $n=1,2,3 \ldots$. Here $\epsilon_{\text {eff }}(q)=\left[\epsilon_{p}+\epsilon_{s} \operatorname{coth}(|q| d)\right] / 2$ is the effective dielectric constant including the gate electrode effect, and $\epsilon_{p}$ and $\epsilon_{s}$ are the dielectric constants of a host piezocrystal and a semiconductor, respectively. ${ }^{23}$

The $n$th harmonic of the SAW potential $\phi_{n}^{\mathrm{SAW}}$ is given only by $a_{n}(x)$ and by material constants and can be easily found from the Poisson equation (10). At $z=d$ we have $\phi_{n}^{\mathrm{SAW}}(x, d)=C_{n} a_{n}(x)$, where the coefficient $C_{n}$ depends on the geometry and the material constants. For example, in a crystal of the type of GaAs, $C_{n}=p_{4} g\left(q_{n}\right)$, where $g\left(q_{n}\right)$ is a complicated function of $q_{n}$. Below, we will give the necessary relations for the hybrid structures.

To find the harmonic amplitudes $n_{n}$ and $\phi_{n}$, we have to solve Eqs. (3), (4), and (5) regarding the "slow" variable $x$ as the constant. The slowly varying quantities $n_{n}(x)$ and $j_{n}(x)$ can be found as Fourier components of the solution $n\left(x_{1}, x\right)$ from a "fast" equation in terms of $x_{1}$. In a selfconsistent approach, the electron density $n\left(x_{1}, x\right)$ is determined by the SAW-induced potential at $z=d$, $\phi^{\mathrm{SAW}}\left(x_{1}, x, d\right)=\phi_{0}^{\mathrm{SAW}}(x, d)+\sum_{n=1,2, \ldots} C_{n} a_{n}(x) e^{i q_{n} x_{1}}+$ c.c., through a nonlinear equation. ${ }^{8}$ By using Poisson's equation, the results of Appendix A, and Eqs. (4) and (5), we obtain 


$$
\begin{aligned}
& |e| n\left(x_{1}, x\right) \mu\left[-\frac{d}{d x_{1}}\left\{\int_{-\infty}^{+\infty} d x_{1}^{\prime} G\left(x_{1}-x_{1}^{\prime}\right) n\left(x_{1}^{\prime}, x\right)\right\}\right. \\
& \left.+E^{\mathrm{SAW}}\left(x_{1}, x\right)\right]-e D_{e} \frac{d n\left(x_{1}, x\right)}{d x_{1}}-e v_{s 0} n\left(x_{1}, x\right)=b_{0},
\end{aligned}
$$

$$
G\left(x_{1}-x_{1}^{\prime}\right)=e \int_{-\infty}^{+\infty} d k \frac{e^{-i k\left(x_{1}-x_{1}^{\prime}\right)}}{|k| \epsilon_{\mathrm{eff}}(|k|)},
$$

where $b_{0}$ is a constant, which occurs after one integration in the conservation-of-charge equation (4). Taking into account $\Gamma^{0} \lambda \ll 1$, we assume that the solution of Eq. (12) is periodic in $\quad x_{1}, \quad n_{s}\left(x_{1}, x\right)=n_{s}\left(x_{1}+\lambda, x\right)$. Also, $\left\langle n_{s}\left(x_{1}, x\right)\right\rangle$ $=\int_{0}^{\lambda} n_{s}\left(x_{1}, x\right) d x_{1} / \lambda=n_{0}(x)$, where $n_{0}(x)$ plays the role of a "local", 2D density.

The functions $a_{n}(x)$ are connected by the system of nonlinear equations (see Appendix B)

$$
\frac{d a_{n}}{d x}=i \delta q_{n}\left[a_{1}, a_{2}, \ldots ; n_{0}, E_{0}\right] a_{n}
$$

where

$$
\begin{aligned}
\delta q_{n}\left[a_{1}, a_{2}, \ldots ; n_{0}, E_{0}\right] & =-\frac{K_{\mathrm{eff}}^{2}\left(q_{n}\right)}{2} \frac{2 \pi}{\epsilon_{\mathrm{eff}}\left(q_{n}\right)} \frac{e n_{n}(x)}{\phi_{n}^{\mathrm{SAW}}(x)} \\
& =i \frac{K_{\mathrm{eff}}^{2}\left(q_{n}\right)}{2 q_{n} \sigma_{m}\left(q_{n}\right)} \frac{j_{n}(x) E_{n}^{\mathrm{SAW} *}}{\left|\phi_{n}^{\mathrm{SAW}}(x)\right|^{2}}
\end{aligned}
$$

and $n=1,2,3, \ldots$.

The local velocity change of a SAW $\delta v_{n}$ and the local absorption coefficient $\Gamma_{n}$ can be expressed by $\delta q_{n}$ :

$$
\delta q_{n}=-q_{n} \frac{\delta v_{n}}{v_{s}^{0}}+i \frac{\Gamma_{n}}{2} .
$$

Using the relation

$$
K_{\mathrm{eff}}^{2}\left(q_{n}\right)=\frac{2\left|E_{n}^{\mathrm{SAW}}\right|^{2} \sigma_{m}\left(q_{n}\right)}{q_{n} I_{n}}
$$

(see Ref. 24) and Eqs. (11) we write

$$
\begin{aligned}
\frac{\delta v_{n}(x)}{v_{s}^{0}} & =\frac{\left\langle\widetilde{\phi}_{n}^{\mathrm{SAW}}\left(x_{1}, x\right) j\left(x_{1}, x\right)\right\rangle}{2 I_{n}(x)}, \\
\Gamma_{n}(x) & =\frac{\left\langle\widetilde{E}_{n}^{\mathrm{SAW}}\left(x_{1}, x\right) j\left(x_{1}, x\right)\right\rangle}{I_{n}(x)} .
\end{aligned}
$$

Here we use the notations $\left\langle f\left(x_{1}, x\right)\right\rangle=\int_{0}^{\lambda} f\left(x_{1}, x\right) d x_{1} / \lambda$, and $\tilde{f}_{n}\left(x_{1}, x\right)=f_{n}(x) \exp \left(i q_{n} x_{1}\right)+$ c.c.

In this section, we have assumed that $K_{\text {eff }}^{2} \ll 1$ and neglected the terms $d^{2} a_{n} / d x^{2} \propto \delta q_{n} d a_{n} / d x \propto K_{\text {eff }}^{4} \quad$ and $d \delta q_{n} / d x \propto K_{\text {eff }}^{4}$. The static electric fields $\phi_{0}(x, z)$ in Eq. 7, which can be induced by a SAW, will not play an important role in this paper because we will consider the case with no voltage applied to the Ohmic contacts $\left(V_{1}=V_{2}\right)$ and a relatively short device with $L \ll 1 / \Gamma^{0}$. Thus, $\phi_{0}(x, d) \simeq$ const and the static electric fields $E_{0 x}(x, d)$ can be neglected. Equations (14) and (15) can now be applied to various types of SAW's by introducing specific electromechanical coupling coefficients.

\section{ACOUSTOELECTRIC TRANSPORT IN A TWO-DIMENSIONAL PLASMA}

Here, we intend to consider the SAW absorption and the acoustic charge transport in the regime $V_{1}=V_{2}$. The behavior of a $2 \mathrm{DEG}$ in an intense SAW at small distances $(\sim \lambda)$ can be assumed to be periodic and is described by nonlinear equation (12). At long distances $(\gg \lambda)$ the SAW behavior is determined by complex amplitudes $a_{n}(x)$, that can be found by Eqs. (14) and (15). First, we define the boundary conditions at $x=0: a_{1}(0)=\sqrt{I_{1}(0)}$ and $a_{n}(0)=0$ for $n=2,3, \ldots$. Here, $I_{1}(0)$ denotes the SAW intensity generated by IDT1. In this case, for a relatively short sample the SAW contains mostly the fundamental SAW harmonic $n=1$ with a small admixture of the higher harmonics $n=2,3, \ldots$. So, it follows from Eqs. (14) and (15)

$$
a_{1}(x)=a_{1}(0)\left(1+i x \delta q_{1}\left[a_{1}(0), 0,0, \ldots ; n_{0}, 0\right]\right) .
$$

We assume that $\left[I_{1}(0)-I_{1}(L)\right] \ll I_{1}(0)$, where $L$ is the length of a semiconductor film and $I_{1}(L)$ is the intensity detected at the IDT2. The latter is valid in a short sample where $\Gamma_{1} L \ll 1$. The first-harmonic absorption coefficient per unit length, which is measured in the experiments, is $\left[I_{1}(0)-I_{1}(L)\right] /\left[I_{1}(0) L\right]=2 \operatorname{Im}\left\{\delta q_{1}\left[a_{1}(0), 0,0, \ldots ; n_{0}, 0\right]\right\}$ $=\Gamma_{1}\left(a_{1}, 0,0, \ldots ; n_{0}, 0\right) \equiv \Gamma_{1}$ and the velocity shift is $\delta v_{1}\left(a_{1}, 0,0, \ldots ; n_{0}, 0\right) / v_{s}^{0}=-\operatorname{Re}\left(\delta q_{1}\left[a_{1}, 0,0, \ldots ; n_{0}, 0\right] / q\right)$ $\equiv \delta v_{1} / v_{s}^{0}$.

The amplitudes of higher harmonics in a short sample turn out to be given by

$$
\begin{aligned}
a_{n}(x) & =i x \lim _{a_{n} \rightarrow 0} a_{n} \delta q_{n}\left[a_{1}(0), 0,0, \ldots a_{n}, \ldots ; n_{0}, 0\right] \\
& =i x \frac{\pi K_{\mathrm{eff}}^{2}\left(q_{n}\right)}{\epsilon_{\mathrm{eff}}\left(q_{n}\right)} \frac{e n_{n}(0)}{C_{n}},
\end{aligned}
$$

where $n=2,3,4, \ldots$. Here, we assume that $\left[I_{1}(0)-I_{1}(L)\right]$ $\gg I_{n}$, which is valid for a short sample where $\Gamma_{1} L \ll 1$, and we take into account that for the strongly nonlinear case $\Gamma_{1}$ $\sim \Gamma_{n}$. The intensity of the $n$ th-harmonic is given by $I_{n}(x)$ $=\left|a_{n}(x)\right|^{2}$ and

$$
\left|C_{n}\right|^{2}=\frac{\left|\phi_{n}^{\mathrm{SAW}}\right|^{2}}{\left|a_{n}\right|^{2}}=\frac{K_{\mathrm{eff}}^{2}\left(q_{n}\right)}{2 q_{n} \sigma_{m}\left(q_{n}\right)}
$$

[see Eq. (17)]. By using Eq. (21) we find the intensities of high harmonics at $x=L$ :

$$
\begin{aligned}
I_{n}(L) & =\lim _{a_{n} \rightarrow 0}\left|a_{n} \delta q_{n}\left[a_{1}, 0,0, \ldots, a_{n}, \ldots ; 0,0\right]\right|^{2} L^{2} \\
& =\pi L^{2}\left(K_{\mathrm{eff}}^{2} / \epsilon_{\mathrm{eff}}\right) q_{n} v_{s}^{0} e^{2}\left|n_{n}(0)\right|^{2} .
\end{aligned}
$$

To calculate $\Gamma_{1}$ and $\delta v_{1}$, we numerically solve Eq. (12) for the parameters $a_{1} \neq 0$ and $a_{n}=0$ when $n=2,3, \ldots$. Then we can find the Fourier components of $n\left(x_{1}, a_{1}\right)$. We now cal- 
culate the electron density $n\left(x_{1}, a_{1}\right)$ in the long wave-length limit $q d \ll 1$. In this limit the function given by Eq. (13) is reduced to $G\left(x_{1}-x_{1}^{\prime}\right)=\left(4 \pi e d / \epsilon_{s}\right) \delta\left(x_{1}-x_{1}^{\prime}\right)$ and Eq. (12) now writes as

$$
\begin{gathered}
|e| n\left(x_{1}, a_{1}\right) \mu\left\{-\frac{4 \pi e d}{\epsilon_{s}} \frac{d n\left(x_{1}, a_{1}\right)}{d x_{1}}+F_{1}^{\mathrm{SAW}} \cos \left(q x_{1}+\chi_{1}\right)\right\} \\
-e D_{e} \frac{d n\left(x_{1}, a_{1}\right)}{d x_{1}}-e v_{s}^{0} n\left(x_{1}, a_{1}\right)-b_{0}=0
\end{gathered}
$$

where the SAW-induced piezoelectric field is taken in the form $E^{\mathrm{SAW}}=F_{1}^{\mathrm{SAW}} \cos \left(q x_{1}+\chi_{1}\right)$, and $\chi_{1}$ is the phase of the first SAW harmonic. For simplicity, we put in the following $\chi_{1}=0$. The constant $b_{0}$ in Eq. (23) is directly connected with the kinetic motion of a SAW and thus vanishes for $v_{s}^{0} \rightarrow 0$. Dividing Eq. (23) by $n$ and then integrating over $x_{1}$ we get

$$
\begin{aligned}
& |e| \mu\left\{-\frac{4 \pi e d}{\epsilon_{s}} n\left(x_{1}\right)+\frac{F_{1}^{\mathrm{SAW}}}{q} \sin \left(q x_{1}\right)\right\}-e D_{e} \ln \left[n\left(x_{1}\right)\right] \\
& =\text { const }_{1}+e v_{s}^{0} x_{1}+b_{0} \int_{0}^{x_{1}} \frac{d x_{1}^{\prime}}{n\left(x_{1}^{\prime}\right)} .
\end{aligned}
$$

The left-hand side of the above equation is periodic and thus the right-hand side should be periodic as well. It implies that $b_{0} \int_{0}^{\lambda} d x_{1}^{\prime} / n\left(x_{1}^{\prime}\right)=-e v_{s}^{0} \lambda$.

A numerical solution of Eq. (23) $n\left(x_{1}\right)$ at $T=300 \mathrm{~K}$ and at various averaged densities $N_{s}=\left\langle n\left(x_{1}\right)\right\rangle$ is shown in the inset of Fig. 3. Here, we use the following parameters $\lambda$ $=33 \mu \mathrm{m}, \mu=5000 \mathrm{~cm}^{2} / \mathrm{V} \mathrm{s}, D=\mu(K T / e), \epsilon_{s}=12.5$, and $v_{s}^{0}=3.8 \times 10^{5} \mathrm{~cm} / \mathrm{s}$. It is seen that with decreasing $N_{s}$ the formerly homogenous $2 \mathrm{DEG}$ turns into moving electron stripes.

It follows from Eqs. (18),(19) that

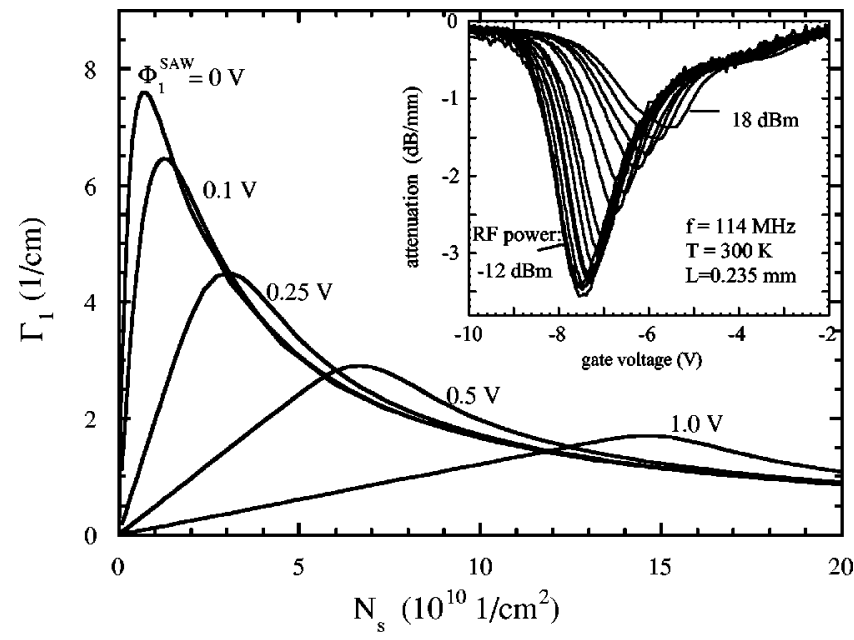

FIG. 2. The calculated absorption coefficient of the first harmonic $\Gamma_{1}$ as a function of the carrier density $N_{s}$ for various potential amplitudes $\Phi_{1}^{\mathrm{SAW}} . \lambda=33 \mu \mathrm{m}, \mu=5000 \mathrm{~cm}^{2} \mathrm{~V} / \mathrm{s}$, and $T$ $=300 \mathrm{~K}$. Inset: The measured attenuation of a SAW as a function of the gate voltage for different high-frequency (rf) powers applied to the IDT1; $f=114 \mathrm{MHz}$.

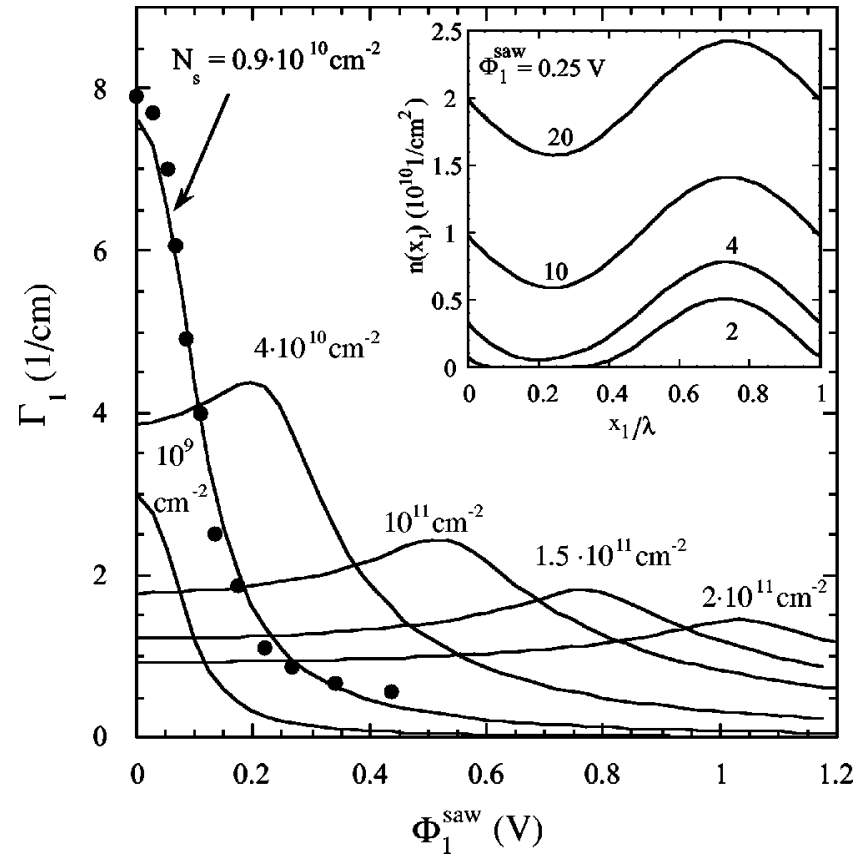

FIG. 3. The calculated absorption coefficient of the first harmonic $\Gamma_{1}$ as a function of the potential amplitude $\Phi_{1}^{\mathrm{SAW}}$ induced by a SAW for various fixed densities $N_{s}$. The parameters are similar to those in Fig. 2. The dots show the experimentally measured absorption coefficient $\Gamma_{1}$ at the gate voltage $-7.5 \mathrm{~V}$. This voltage corresponds to the maximal attenuation at the smallest rf power. In the inset we plot the calculated local carrier concentration $n$ as a function of the in-plane coordinate $x_{1}$ for different total carrier concentration $N_{s}$. The numbers attached to the plots correspond to $N_{s}$ in units of $10^{10} \mathrm{~cm}^{-2}$.

$$
\begin{aligned}
\frac{\delta v_{1}}{v_{s}^{0}} & =-\frac{\left\langle\Phi_{1}^{\mathrm{SAW}} \sin \left(q x_{1}\right) j\left(x_{1}\right)\right\rangle}{2 I_{1}} \\
\Gamma_{1} & =\frac{\left\langle F_{1}^{\mathrm{SAW}} \cos \left(q x_{1}\right) j\left(x_{1}\right)\right\rangle}{I_{1}} .
\end{aligned}
$$

The calculated absorption coefficient $\Gamma_{1}$ as a function of the electron density $N_{s}$ for various potential amplitudes $\Phi_{1}^{\mathrm{SAW}}$ $=F_{1}^{\mathrm{SAW}} / q$ is shown in Fig. 2. It was calculated from Eqs. (25) and the numerical solution for $n\left(x_{1}\right)$. The potential amplitude $\Phi_{1}^{\mathrm{SAW}}$ can be easily connected with the SAW intensity $I_{1}$ by using Eqs. 17 and 22 with $K_{\text {eff }}^{2}$ found numerically in Ref. 9. As an example, in the hybrid structures at SAW frequencies $f=114$ and $340 \mathrm{MHz}, K_{\text {eff }}^{2}$ is about 0.015 and 0.035 , respectively.

We see in Fig. 2 that with increasing $\Phi_{1}^{\mathrm{SAW}}$ the absorption coefficient in general decreases and its maximum is shifted to the higher values of $N_{s}$. This nonlinear behavior can be understood qualitatively as follows. For the densities $N_{s} \ll N_{s}^{\max }$, the electron plasma forms the moving charge stripes and the electron velocity $j /\left(e N_{s}\right)$ is very close to its maximum $v_{s}^{0}$. Here $N_{s}^{\max }$ denotes the density corresponding to the maximum of the function $\Gamma_{1}\left(N_{s}\right)$ at fixed $\Phi_{1}^{\mathrm{SAW}}$. We will show below that in the case $N_{s}<N_{s}^{\max } \Gamma_{1}$ $\simeq|e| N_{s}\left(v_{s}^{0}\right)^{2} /\left(I_{1} \mu\right) \propto N_{s} / I_{1}$. This asymptotic behavior follows from the Weinreich relation. ${ }^{25}$ In the region $N_{s} \gg N_{s}^{\max }$, 


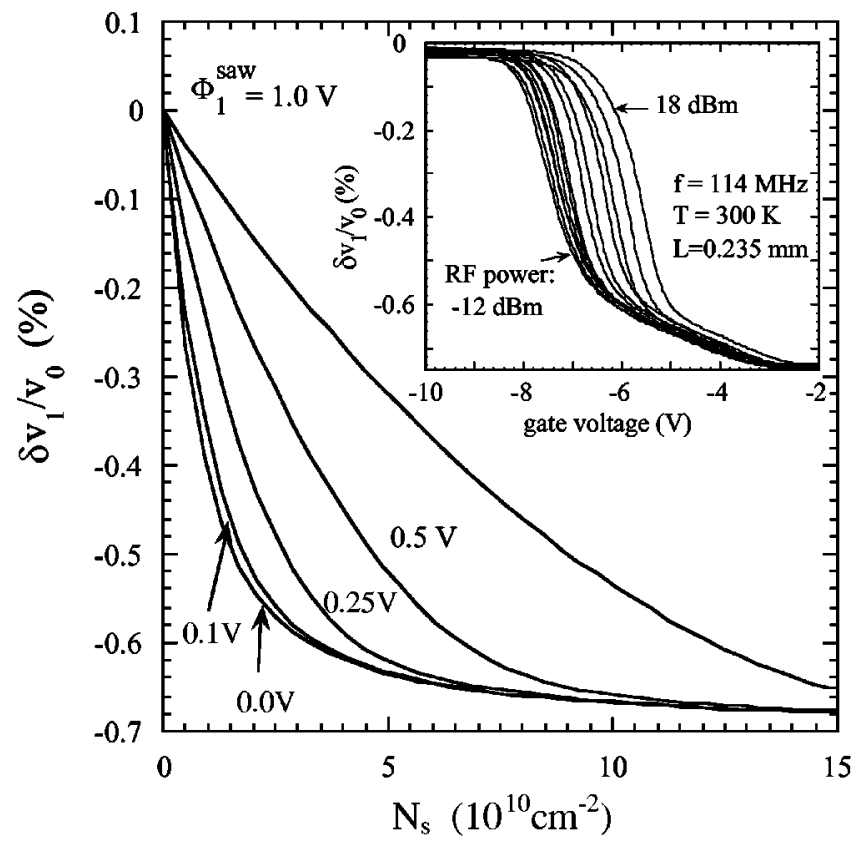

FIG. 4. The calculated SAW-velocity change $\delta v_{1}$ as a function of the electron density for various potential amplitudes $\Phi_{1}^{\mathrm{SAW}}$. The parameters are similar to those in Fig. 2. Inset: The measured velocity change of a SAW as a function of the gate voltage for different high-frequency (rf) powers applied to the IDT1; $f$ $=114 \mathrm{MHz}$.

the electric current $\sigma E_{x}$ decreases with increasing $N_{s}$ because of the screening effect. Thus, the absorption coefficient decreases as well.

In Fig. 3 we plot $\Gamma_{1}$ as a function of the SAW potential $\Phi_{1}^{\text {SAW }}$ for a fixed $2 D E G$ density. For electron densities less than about $10^{10} \mathrm{~cm}^{-1}$, the function $\Gamma_{1}\left(\Phi_{1}^{\mathrm{SAW}}\right)$ is always decreasing. At higher densities $\Gamma_{1}\left(\Phi_{1}^{\mathrm{SAW}}\right)$ has a maximum. We attribute this behavior to the screening effect in a $2 \mathrm{DEG}$. At high density and small $\Phi_{1}^{\mathrm{SAW}}$, the absorption is strongly suppressed because of screening. The intense SAW, however, modulates the 2DEG and, consequently, reduces screening. Thus, the absorption coefficient starts to increase with increasing $\Phi_{1}^{\mathrm{SAW}}$, when $\Phi_{1}^{\mathrm{SAW}}$ is not so large. At larger $\Phi_{1}^{\text {SAW }}$, the plasma becomes broken into stripes and $\Gamma_{1}$ decreases with $I_{1}$ as $1 / I_{1} \cdot{ }^{8,18}$

The velocity shift $\delta v_{1}$ numerically calculated by Eqs. (25) is shown in Fig. 4. With increasing SAW intensity $\delta v_{1}$ increases and approaches to the velocity $v_{s}^{0}$ that corresponds to the case of a totally depleted 2DEG. By analyzing Eqs. (25) and the function $j\left(x_{1}\right)$, we can see that, in the limit $I_{1} \rightarrow \infty$, $\delta v_{1} \propto 1 / \Phi_{1}^{\mathrm{SAW}} \propto 1 / \sqrt{I_{1}}$, as in a $3 \mathrm{D}$ plasma. ${ }^{12}$

In Fig. 5 we show results of numerical calculations for the intensities of the high harmonics $I_{n} / I_{n, \max }=\left|n_{n}\right|^{2} / N_{s}^{2}$, where

$$
I_{n, \max }=I_{n}\left(\Phi_{1}^{\mathrm{SAW}} \rightarrow \infty\right)=\pi L^{2} \frac{K_{\mathrm{eff}}^{2}}{\epsilon_{\mathrm{eff}}} q_{n} v_{s}^{0} e^{2} N_{s}^{2} .
$$

This formula was obtained taking into account that $\left|n_{n}\right|$ $\rightarrow N_{s}$ when $\Phi_{1}^{\mathrm{SAW}} \rightarrow \infty$. The quantities $I_{n}$ were calculated from the Fourier components of the function $n\left(x_{1}\right)$. At small amplitudes $\Phi_{1}^{\mathrm{SAW}}, I_{n} \propto\left(\Phi_{1}^{\mathrm{SAW}}\right)^{n}$, being typical for weak nonlinearity. At larger $\Phi_{1}^{\mathrm{SAW}}$, the behavior of $I_{n}$ is quite com-

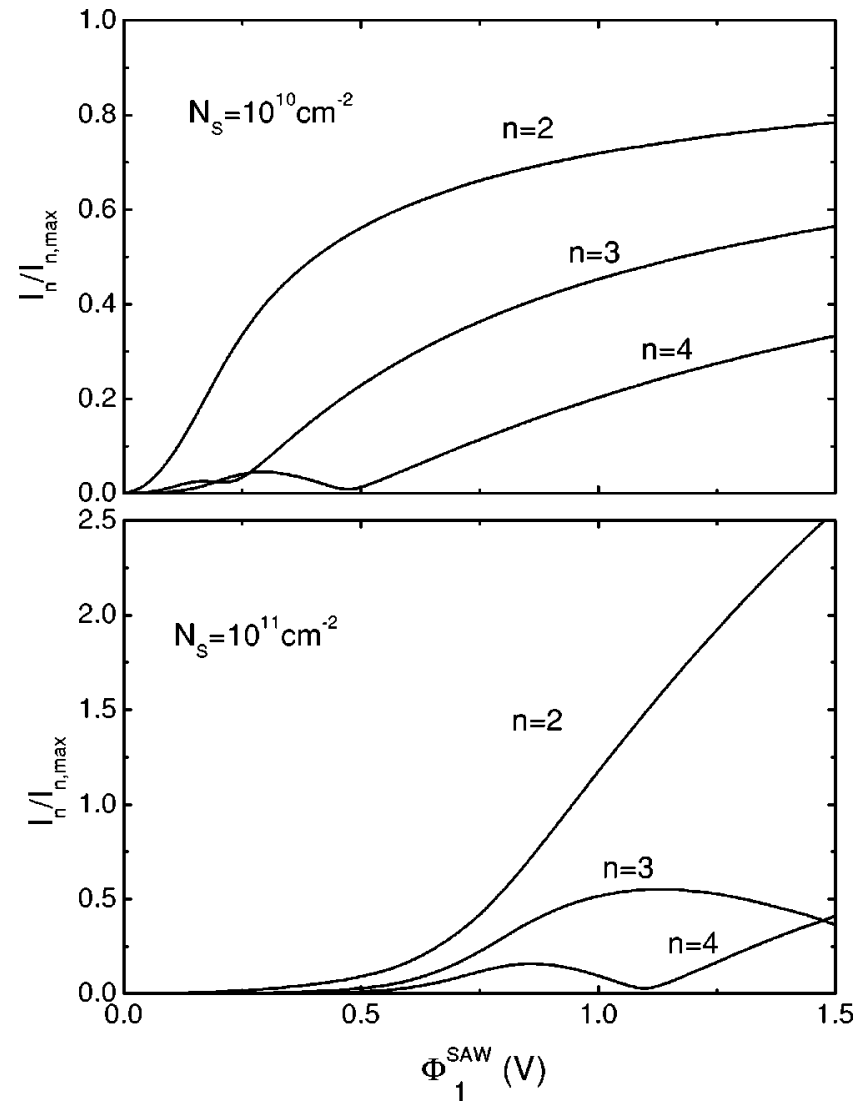

FIG. 5. The calculated intensities of higher harmonics with $n$ $=2,3$, and 4 as functions of the potential amplitude $\Phi_{1}^{\mathrm{SAW}}$ for two electron densities $N_{s}=10^{10} \mathrm{~cm}^{-2}$ (upper part) and $N_{s}=10^{11} \mathrm{~cm}^{-2}$ (lower part). The parameters are similar to those in Fig. 2.

plex and strongly differs from the weak-nonlinearity behavior. At very large $\Phi_{1}^{\mathrm{SAW}}$, the high-harmonics intensities tends to saturate: $I_{n} \rightarrow I_{n \text { max }}$. At higher electron densities the saturation of $I_{n}$ occurs at larger SAW potentials (see Fig. 5).

\section{ANALYTIC RESULTS FOR ACOUSTOELECTRIC EFFECTS IN A 2D ELECTRON PLASMA}

In this section, we will give some analytic expressions describing various acoustoelectric effects in a system with a 2DEG. For simplicity, we will not take into account diffusion, hence assuming $D_{e}=0$ in the formula for the current (5). This assumption is well justified at large electron densities or at low temperatures. It is convenient to start with the Weinreich relation for a $2 \mathrm{D}$ system in the nonlinear regime. ${ }^{12,25}$ Assuming that $n\left(x_{1}\right)$ and $j\left(x_{1}\right)$ are periodic functions we can rewrite Eq. (12) in the form

$$
j\left(x_{1}\right)-v_{s}^{0} e n\left(x_{1}\right)=b_{0} .
$$

Obviously, it is valid in the limit $K_{\text {eff }}^{2} \ll 1$. Using Eq. (26) we can write $\langle j\rangle=|e| \mu\left\langle n E_{x}\right\rangle=-\mu\left\langle j E_{x}\right\rangle / v_{s}^{0}$. From this equation we now get the Weinreich relation

$$
\frac{\left\langle j E_{x}\right\rangle}{\langle j\rangle}=-\frac{v_{s}^{0}}{\mu}
$$


where $\left\langle j E_{x}\right\rangle$ is the dissipation in a SAW. To get Eq. (27), we have neglected the averaged electric fields $\left\langle E_{x}\right\rangle=0$ assuming the case $V_{1}=V_{2}$.

We now consider Eq. 23 with $D_{e}=0$. At fixed $\Phi_{1}^{\mathrm{SAW}}$, there is a critical density $N_{\text {crit }}$, for the formation of stripes in a 2DEG. When $N_{s}<N_{\text {crit }}$ the plasma is split into electron stripes. If $N_{s}>N_{\text {crit }}$, the plasma is continuous but can be strongly modulated in space. For the case $N_{s}<N_{\text {crit }}$, the constant $b_{0}$ in Eq. (23) becomes zero and thus Eq. (23) has formally two solutions

$$
\begin{gathered}
f_{1}\left(x_{1}\right)=\text { const }_{2}-\frac{\epsilon_{s} \Phi_{1}^{\text {SAW }}}{4 \pi|e| d} \sin \left(q x_{1}\right)-\frac{v_{s}^{0}}{\mu} \frac{\epsilon_{s}}{4 \pi|e| d} x ; \\
f_{2}\left(x_{1}\right)=0 .
\end{gathered}
$$

The solution has to be a periodic continuous combination of these two functions. It follows from Eq. (28) that in the limit $\sigma_{0} \gg \sigma_{m} N_{\text {crit }} \simeq \Phi_{1}^{\mathrm{SAW}} \epsilon_{s} /(4 \pi|e| d)$, where $\sigma_{0}=|e| \mu N_{s}$.

When $N_{s}<N_{\text {crit }}$ the plasma turns into stripes, which means that electrons are totally trapped and the local electron velocity in a 2DEG reaches its maximum $j /\left(e N_{s}\right)=v_{s}^{0}$. From the Weinreich relation, we get $\Gamma_{1}=\left\langle j E_{x}\right\rangle / I_{1}$ $=|e| N_{s}\left(v_{s}^{0}\right)^{2} /\left(\mu I_{1}\right) \propto 1 / I_{1} \cdot{ }^{8,16}$

Equations (23) and (24) can be used to find an asymptotic formula for $\Gamma_{1}$ and $\langle j\rangle$ in the large density limit when $\sigma_{0} \gg \sigma_{m}$. In the limit $\sigma_{0} \gg \sigma_{m}, n \simeq N_{s}$ $-\left(\epsilon_{s} \Phi_{1}^{\mathrm{SAW}}\right) /(4 \pi|e| d) \sin \left(q x_{1}\right)$ [see Eq. (28)]. By using the above and Eqs. (26) and (27), we have in the limit $\sigma_{0} \gg \sigma_{m}$ and in the region $\Phi_{1}^{\mathrm{SAW}}<\Phi_{\text {crit }}$ :

$$
\Gamma_{1}=\Gamma_{\max } \frac{4 \sigma_{m}}{\sigma_{0}}\left(\frac{\Phi_{\text {crit }}}{\Phi_{1}^{\mathrm{SAW}}}\right)^{2}\left(1-\sqrt{1-\left[\frac{\Phi_{1}^{\mathrm{SAW}}}{\Phi_{\text {crit }}}\right]^{2}}\right),
$$

where $\Gamma_{\max }=q K_{\mathrm{eff}}^{2} / 2$ and $\Phi_{\text {crit }}=N_{s} 4 \pi|e| d / \epsilon_{s}$. This equation is valid when $\left(\Phi_{\text {crit }}-\Phi_{1}^{\mathrm{SAW}}\right) / \Phi_{\text {crit }} \gg \sigma_{m} / \sigma_{0}$. Equation (29) reproduces the numerical data for $\Gamma_{1}\left(\Phi_{1}^{\mathrm{SAW}}\right)$ in Fig. 3 at large densities. The asymptotic formula (29) was given before in Ref. 26 without noting the condition $\sigma_{0} \gg \sigma_{m}$. In the linear regime of interaction $\Gamma_{1}$ and $\delta v_{1}$ are given by the formula (1).

In the end of this section we consider an asymptotic behavior for the high-harmonic intensities $I_{n}$ in the limit $I_{1}$ $\rightarrow \infty$. The electron density $n_{s}\left(x_{1}\right)$ at high $\Phi_{1}^{\text {SAW }}$ can be written in a parabolic approximation. Then, by calculating the Fourier components $n_{n}$, we find that $I_{n, \max }-I_{n}$ $\propto\left(\Phi_{1}^{\mathrm{SAW}}\right)^{-2 / 3} \propto I_{1}^{-1 / 3}$, where $n=2,3, \ldots$.

\section{COMPARISON WITH EXPERIMENTAL DATA}

The experiments involving SAW's were performed on the hybrid semiconductor-LiNbO ${ }_{3}$ structures fabricated by the epitaxial lift-off (ELO) technique developed by Yablonovich et $a .^{27}$ The structures contain a 12-nm-thick high-quality $\mathrm{In}_{0.2} \mathrm{Ga}_{0.8} \mathrm{As}$ quantum well $(\mathrm{QW})$ embedded in modulation doped $\mathrm{Al}_{0.2} \mathrm{Ga}_{0.8} \mathrm{As}$ barriers. In these structures, the thin semiconductor layered system including a QW was tightly bound to the lithium niobate host crystal by the van der Waals forces. ${ }^{8-10}$ The MBE grown quantum well structure is removed from its native GaAs substrate by etching an AlAs sacrificial layer below the active semiconductor system. The thin ELO film with a thickness of only $500 \mathrm{~nm}$ is then transferred onto the host $\mathrm{LiNbO}_{3}$ crystal. The parameters for this structure were described already in the beginning of Sec. I. The geometry of the structure is shown in Fig. 1. For further details related to the fabrication procedure of such quasimonolithic structures we refer the reader to Refs. 8-10. The experiments were performed for two SAW frequencies $f$ $=340 \mathrm{MHz}$ and $f=114 \mathrm{MHz}$ at room temperature.

The SAW in our experiments can be strong enough to break up an initially homogenous 2D plasma into moving stripes. The transition to the regime of moving electron stripes was directly observed in the experiments on acoustic charge transport (ACT) in samples with specially designed injection and detection dates. ${ }^{8}$ In these experiments the velocity of the ACT signal first increases with the SAW intensity and finally saturates at the sound velocity. The latter manifests the formation of stripes. Strongly nonlinear effects are also observed in the attenuation data. The attenuation of a SAW with $f=114 \mathrm{MHz}$ for different intensities is plotted in the inset of Fig. 2 as a function of the transport-gate voltage, which determines the averaged electron density in a 2DEG. At small SAW intensities, the electronic sound attenuation $\Gamma^{0}$ as a function of the conductivity $\sigma_{0}$ is described by the well-known linear-theory equation (1) and exhibits a maximum. This linear regime is realized in our experiments at the smallest SAW intensities of about $-12 \mathrm{dBm}$ (inset of Fig. 2). It is seen from the inset of Fig. 2 that at high SAW amplitudes the attenuation is strongly suppressed and its maximum is shifted to higher gate bias or conductivity, respectively. The experimental data for a SAW with the frequency $f=340 \mathrm{MHz}$ look qualitatively similar to those for $f=114 \mathrm{MHz}$ and were given earlier in Ref. 8 .

The nonlinear regime of interaction is described by the theory given in Secs. II, III, and IV. To quantitatively compare theory and experiment, we now express the SAW potential amplitude $\Phi_{1}^{\mathrm{SAW}}$ through the input radio frequency (rf) power $P$. The SAW intensity can be written as $I_{1}$ $=I_{\mathrm{SAW}}=2(P / w) 10^{-I L / 10}$, where the width of the transducer $w=0.55 \mathrm{~mm}$. The insertion losses (IL) in the transducers were measured to be $15 \mathrm{~dB}$. Then, the $\mathrm{SAW}$ potential can be written using Eq. (22) as $\Phi_{1}^{\mathrm{SAW}}=K_{\text {eff }} \sqrt{2 I_{1} /\left(q \sigma_{m}\right)}$, where $K_{\text {eff }}^{2}=0.015$ for $f=114 \mathrm{MHz} .{ }^{10}$ In Fig. 3 we also show the experimentally measured absorption coefficient at the gate voltage $V_{t}=-7.5 \mathrm{~V}$. This voltage corresponds to the maximal attenuation for the smallest $\mathrm{rf}$ power, $-12 \mathrm{dBm}$ (see inset of Fig. 2). From the linear theory we find that the absorption coefficient is maximal at $N_{s}=0.9 \times 10^{10} 1 / \mathrm{cm}^{2}$. One can see from Fig. 3 that the experimentally measured function $\Gamma_{1}\left(\Phi_{1}^{\mathrm{SAW}}\right)$ for $V_{t}=-7.5 \mathrm{~V}$ is in very good agreement with the calculated one for $N_{s}=0.9 \times 10^{10} \mathrm{~cm}^{-2}$. Here we did not use any fitting parameters. This quantitative agreement becomes possible if we account for the diffusion coefficient. The maximal absorption coefficient as calculated from the linear theory is $\Gamma_{\max }^{\text {diff }}=7.6 \mathrm{~cm}^{-1}$. Without diffusion this value is about $\Gamma_{\max }=14.3 \mathrm{~cm}^{-1}$. Thus, the diffusion strongly suppresses the SAW absorption.

At fixed SAW power and a sufficiently small density $N_{s}$, the 2DEG is divided into stripes and $\Gamma_{1}$ increases with increasing the gate voltage. In our theory, $\Gamma_{1} \propto N_{s}$ in the regime 


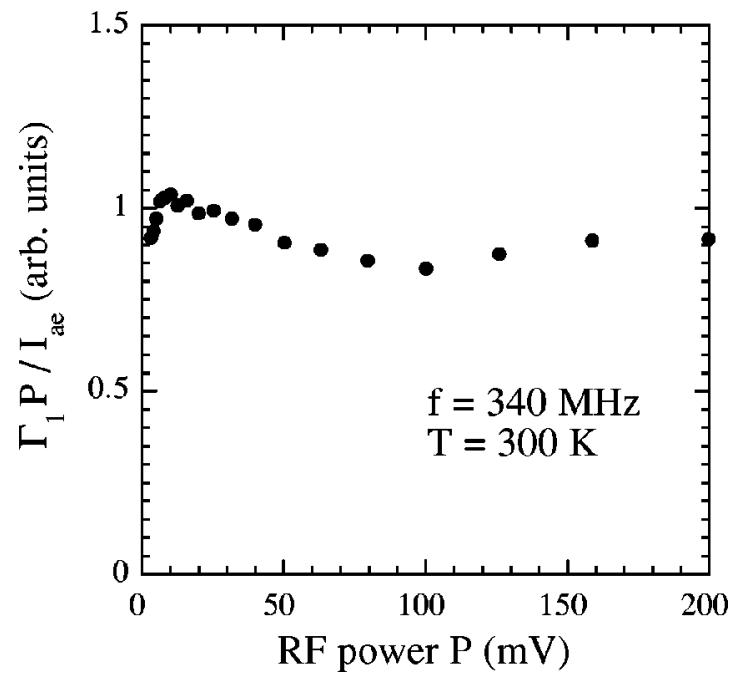

FIG. 6. The measured ratio $\Gamma_{1} P / I_{a e}$ as a function of the high-frequency (rf) power $P$ for $f=340 \mathrm{MHz} ; T=300 \mathrm{~K}$. The acoustoelectric current $I_{\mathrm{AE}}$ was measured in its maximum.

of stripes, which explains the increase of the attenuation at small gate voltages in the inset of Fig. 2. At a sufficiently large gate voltage, the absorption coefficient as a function of $V_{t}$ starts to decrease because of the screening effect in a high-density $2 \mathrm{DEG}$ modulated by a SAW. The interplay of these two effects leads to the shift of the maximum of the function $\Gamma_{1}\left(V_{t}\right)$ shown in the inset of Fig. 2.

The change of the SAW velocity due to the electron plasma is shown in the inset of Fig. 4. With increasing rf power the curves in Fig. 4 are again shifted towards larger electron conductivity which can be understood in terms of screening. With increasing SAW intensity the electron plasma is strongly modulated or even split into stripes and the screening of piezoelectric fields by electrons becomes not so effective. Thus, the shift of the SAW velocity due to electrons decreases with increasing the SAW intensity. The experimentally observed shift is in qualitative agreement with our modeling shown in Fig. 4. However, our theory does not reproduce the character of $\delta v_{1}\left(V_{t}\right)$ in the region of small electron densities. Likely, the measurement of the SAW velocity is not so sensitive to a low-density electron system in comparison with the attenuation method.

In Fig. 6, we show the quantity $\Gamma_{1} P / I_{a e}$ as a function of the rf power to verify the Weinreich relation. The acoustoelectric current $\langle j\rangle=I_{\mathrm{AE}}(P)$ was measured in a "short circuit geometry,' where the Ohmic contacts are directly (without resistor) connected to the current measurement instrument. For more details on acoustoelectric current measurements we refer to Ref. 11. We see from Fig. 6 that the ratio $\Gamma_{1} P / I_{\mathrm{AE}}$ has a weak power dependence. Thus, our experimental data are well described by the Weinreich relation. Slight deviations from the Weinreich relation seen in Fig. 6 can come from the density dependence of the mobility $\mu\left(N_{s}\right)$, that is expected to be relatively weak at room temperature. The reason is that the main electronic scattering mechanism at high temperatures is due to acoustic phonons and is relatively insensitive to the $2 \mathrm{D}$ density.

\section{CONCLUSIONS}

The theoretical results obtained in Sec. II, can also be applied to study dynamics of SAW's at large distances in a long sample, where the contribution of high harmonics can be very important. ${ }^{28,29}$ This long-distance transformation to high harmonics was studied experimentally for SAW's interacting with a 3D electron gas of a semiconductor on a piezocrystal. $^{28,29}$ In the presence of a dc voltage applied to the crystal, it is possible to expect the appearance of nonlinear waves with a stationary profile or with a stationary energy flow. ${ }^{12,14,28}$ In a wave with a stationary energy flow the wave shape is periodically changed in space. ${ }^{28}$ Another scenario can relate to chaotic dynamics in an acoustoelectric system. ${ }^{30}$ Equation (14) can be used to numerically model these phenomena in 2D electron systems at long distances.

To conclude, we have studied strongly nonlinear acoustoelectric phenomena caused by the interaction between a SAW and a two-dimensional electron system. In the experimental measurements performed on hybrid semiconductorpiezocrystal structures the SAW attenuation, the SAW velocity change, and the acoustoelectric current are strongly modified in the nonlinear regime due to the formation of moving electron stripes. By using a coupled-amplitude method we have modeled the decay and transformation of SAW's in the nonlinear regime. Using our theoretical results, we could explain our experimental findings and distinguished between different regimes of the nonlinear acoustoelectric interaction at large SAW intensities.

\section{ACKNOWLEDGMENTS}

We would like to thank D. Bernklau and H. Riechert for the fabrication of the excellent MBE material, W. Ruile for the strong support from the SAW device side, and A. V. Chaplik and M. K. Balakirev for helpful discussions. We gratefully acknowledge financial support by the Volkswagen-Stiftung and by the Russian Foundation for Basic Research (Grants No. 99-02-17019 and 99-02-17127).

\section{APPENDIX A}

Here we intend to briefly discuss the electrostatics of the hybrid structure. The spacing between the 2DEG and the top metal gate in the fabricated structures is much larger than the distance from the $2 \mathrm{DEG}$ to the $\mathrm{AlGaAs}-\mathrm{LiNbO}_{3}$ interface. Thus, to model the screening effects, we will assume that the

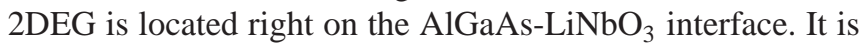
convenient to solve this problem by a Fourier transform in terms of $x$ and by remaining the vertical coordinate $z$. The relation between the Fourier components of the electrostatic potential $W^{\text {ind }}[z ; k]$ induced by $2 \mathrm{D}$ electrons and the $2 \mathrm{DEG}$ density $n_{k}$ is found from the Poisson equation and from the corresponding boundary conditions

$$
W^{\text {ind }}[z ; k]=\frac{2 \pi e n_{k}}{|k| \epsilon_{\text {eff }}(k)} G(z),
$$

where $\epsilon_{\text {eff }}(k)=\left[\epsilon_{p}+\epsilon_{s} \operatorname{coth}(|k| d)\right] / 2$. The function $G(z)$ $=\sinh |k| z / \sinh |k| d$, when $0<z<d$, and $G(z)=e^{-|k|(z-d)}$ for $d<z^{23}$

Taking the electron density in the form of $n_{s}\left(x, x_{1}, t\right)$ $=n_{0}(x)+\sum_{n=1,2, \ldots} n_{n}(x) e^{i q_{n} x_{1}}+$ c.c., we can write for the induced electrostatic potential $\phi^{\text {ind }}\left(x, x_{1}, z, t\right)=\phi_{0}^{\text {ind }}(x, z)$ $+\sum_{n=1,2, \ldots} \phi_{n}^{\text {ind }}(x, z) e^{i q_{n} x_{1}}+$ c.c., where $q_{n}=n q$. Using Eq. $(\mathrm{A} 1)$, we find $\phi_{n}^{\text {ind }}(x, z)=2 \pi e n_{n}(x) G(z) /\left[q_{n} \epsilon_{\mathrm{eff}}\left(q_{n}\right)\right]$ 
$+\delta \phi_{n}(x, z)$, where $n=1,2, \ldots$. The correction $\delta \phi_{n}(x, z)$ $\sim d a_{n}(x) / d x \sim \delta q_{n} \sim K_{\text {eff }}^{2}$ [see Eq. (15) for $\delta q_{n}$ ] and is small compared to $W^{\text {ind }}\left[z ; q_{n}\right]$. Thus, regarding $n_{n}(x)$ as the constants, we obtain at $z=d$ : $\phi_{n}^{\text {ind }}(x, d)$ $\simeq 2 \pi e n_{n}(x) /\left[q_{n} \epsilon_{\mathrm{eff}}\left(q_{n}\right)\right]$ and $E_{n x}(x, d) \simeq-i q_{n} \phi_{n}^{\text {ind }}(x, d)$. In other words, we consider the envelope functions $n_{n}(x)$ as constants and solve Poisson's equation in terms of the "fast", variable $x_{1}$. Again, it is valid in the limit $\Gamma^{0} / q$ $\sim K_{\text {eff }}^{2} \ll 1$.

As an example, we now calculate the correction $\delta \phi_{n}$ in the linear regime of interaction, when $n\left(x, x_{1}, t\right)=N_{s}+n_{1}(x) e^{i q x_{1}}+n_{1}^{*}(x) e^{-i q x_{1}} \quad$ with $n_{1}(x)$ $=\tilde{n}_{1} e^{-\Gamma^{0} x / 2-i q \delta v_{s}^{0} / v_{s}^{0} x}$. From the Poisson equation we find $\delta \phi_{1}(x, z) \sim\left(\Gamma^{0} / 2+i q \delta v_{s} / v_{s}^{0}\right) n_{1}(x) F(z)$, where $F(z) \sim 1$ for $z \sim 1 / q$.

\section{APPENDIX B}

In order to solve a system of nonlinear equations (2)-(5) we will use some of results from a linear-response theory. ${ }^{20,21}$ In a linear theory the total electrostatic potential and the 2D density can be written as $W[x, z, t]$ $=W[z, k] e^{i k x-i \omega t}$ and $n(x, t)=n_{k} e^{i k x-i \omega t}$, respectively. Here $\omega$ is the SAW frequency. It is convenient to introduce a quantity $\Pi_{k}$ by means of the relation $e n_{k}=-\Pi_{k} W[d ; k]$, where $W[d, k]$ is the Fourier component of the electrostatic potential at $z=d$. Equations (2), (3) are now written as

$$
\begin{gathered}
\omega^{2} \rho u_{i}+c_{i k l m} \partial_{m} \partial_{k} u_{l}+p_{l i k} \partial_{l} \partial_{k} W[x, z, t]=0, \\
-4 \pi p_{i k l} \partial_{i} \partial_{l} u_{k}+\left(\epsilon \partial_{i} \partial_{i}-4 \pi \Pi_{k} \delta\left(x_{3}-d\right)\right) W[x, z, t]=0 .
\end{gathered}
$$

Above equations should be solved together with the necessary boundary conditions considered in Sec. I. Then, we rewrite Eqs. (B1), (B2) in the form

$$
\hat{L}_{\text {lin }} \mathbf{A}_{\text {lin }}=0
$$

Here $\hat{L}_{\text {lin }}$ is a linear operator and $\mathbf{A}_{\operatorname{lin}}=(\mathbf{u}[x, z, t], W[x, z, t])$ $=\left(\mathbf{U}^{0}[z ; k], W^{0}[z ; k]\right) e^{i k x-i \omega t}$. It follows from the boundary conditions that $k=q+\delta q_{\text {lin }},{ }^{21}$ where $q=\omega / v_{s}^{0}$ and

$$
\delta q_{\text {lin }}=\frac{q K_{\mathrm{eff}}^{2}}{2} \frac{\Pi_{q} / \Pi_{q}^{0}}{1+\Pi_{q} / \Pi_{q}^{0}}, \quad \Pi_{q}^{0}=\frac{q \epsilon_{\mathrm{eff}}(q)}{2 \pi} .
$$

Now a solution of Eq. (B3) can be written as $\mathbf{A}_{\text {lin }}=\mathbf{A}^{0}[z ; q$ $\left.+\delta q_{\operatorname{lin}}\right] f_{0}(x) e^{i q x_{1}}$, where $f_{0}(x)=e^{i \delta q_{\operatorname{lin}^{x}}}$ and $x_{1}=x-v_{s}^{0} t$. $\mathbf{A}^{0}\left[z ; q+\delta q_{\text {lin }}\right]$ is a vector, that can be found from the matrix given by the boundary conditions. ${ }^{21}$

*E-mail: govor@isp.nsc.ru

${ }^{1}$ M. J. Hoskins, H. Morko, and B. J. Hunsinger, Appl. Phys. Lett. 41, 332 (1982); W. J. Tanski, S. W. Merritt, R. N. Sacks, D. E. Cullen, E. J. Branciforte, R. D. Caroll, and T. C. Eschrich, ibid. 52, 18 (1988).

${ }^{2}$ A. Wixforth, J. Scriba, M. Wassermeier, J. P. Kotthaus, G. Weimann, and W. Schlapp, Phys. Rev. B 40, 7874 (1989).

${ }^{3}$ R. L. Willett, R. R. Ruel, K. W. West, and L. N. Pfeiffer, Phys. Rev. Lett. 71, 3846 (1993).
Equation B3 contains first and second spatial derivatives and can be written as $\hat{L}_{\text {lin }} \mathbf{A}_{\text {lin }}=e^{i q x_{1}}\left[f_{0}(x) \hat{L}_{0}(q) \mathbf{A}^{0}\right.$ $\left.+f_{0}^{\prime}(x) \hat{L}_{1}(q) \mathbf{A}^{0}+f_{0}^{\prime \prime}(x) \hat{L}_{2}(q) \mathbf{A}^{0}\right]=0$, where $f^{\prime}=d f / d x$. Neglecting the second derivative $f_{0}^{\prime \prime}(x)$, that is $\sim \delta q_{\text {lin }}^{2}$ $\sim K_{\text {eff }}^{4}$, we have

$$
\hat{L}_{0}(q) \mathbf{A}^{0}+i \delta q_{\operatorname{lin}} \hat{L}_{1}(q) \mathbf{A}^{0}=0
$$

Now we turn to the nonlinear theory, where the equation $\hat{L} \mathbf{A}=0$ is also valid. The operator $\hat{L}$ is determined by the equations similar to Eqs. (B1)-(B3) but with nonlinear quantity $\Pi_{n}(x)$, that is defined by $\Pi_{n}(x)=-e n_{n}(x) / \phi_{n}(x, d)$. In the nonlinear case the vector $\mathbf{A}=\mathbf{A}_{0}(x, z)$ $+\sum_{n=1,2, \ldots} a_{n}(x) \mathbf{A}_{n}(x, z) e^{i q_{n} x_{1}}+$ c.c.. Each term in the equation $\hat{L} \mathbf{A}=0$ should be zero, and so $\hat{L} a_{n}(x) \mathbf{A}_{n}(x, z) e^{i q_{n} x_{1}}$ $\simeq e^{i q_{n} x_{1}}\left[a_{n}(x) \hat{L}_{0}\left(q_{n}\right) \mathbf{A}_{n}+a_{n}^{\prime}(x) \hat{L}_{1}\left(q_{n}\right) \mathbf{A}_{n}\right]=0$. To get the latter equation, we have neglected $d \delta q_{n} / d x \sim K_{\text {eff }}^{4}$ and $d^{2} a_{n} / d x^{2} \sim K_{\text {eff }}^{4}$. We can solve the equation $\hat{L} \mathbf{A}=0$ if we choose $\mathbf{A}_{n}(x, z)=\mathbf{A}^{0}\left[z, q_{n}+\delta q_{n}(x)\right]$, where the vector $\mathbf{A}^{0}[z ; q]$ is defined above in the linear theory. Using Eq. (B5) we get

$$
\frac{d a_{n}(x)}{d x}=i \delta q_{n}(x) a_{n}(x)
$$

where $\delta q_{n}(x)$ is given by the equation for $\delta q_{\text {lin }}$ [Eq. (B4)] with corrections $\Pi_{q} \rightarrow \Pi_{n}(x)$ and $\Pi_{q}^{0} \rightarrow \Pi_{q_{n}}^{0}=\Pi_{n}^{0}$. Equation B6 is used in Secs. I and II to describe the acoustoelectric phenomena in a 2DEG. Using Eqs. (8) and (11) and the results of Appendix A, the denominator in $\delta q_{n}(x)$ [see Eq. (B4)] is rewritten as $1+\Pi_{n}(x) / \Pi_{n}^{0}$ $=-\Pi_{n}(x) \phi_{n}^{\mathrm{SAW}}(x) / e n_{n}(x)$. Then, by using Eq. (B4) and the conservation-of-charge equation $j_{n}(x)=v_{s}^{0} e n_{n}(x)$, we obtain

$$
\begin{aligned}
\delta q_{n}(x) & =-\frac{K_{\mathrm{eff}}^{2}\left(q_{n}\right)}{2} \frac{2 \pi}{\epsilon_{\mathrm{eff}}\left(q_{n}\right)} \frac{e n_{n}(x)}{\phi_{n}^{\mathrm{SAW}}(x)} \\
& =i \frac{K_{e f f}^{2}\left(q_{n}\right)}{2 \Pi_{n}^{0} v_{s}^{0}} \frac{j_{n}(x) E_{n}^{\mathrm{SAW} *}}{\left|\phi_{n}^{\mathrm{SAW}}(x)\right|^{2}} .
\end{aligned}
$$

We use this equation in Sec. II [see Eq. (15)]. To obtain above results, we have neglected the terms like $a_{n}^{\prime \prime}(x)$ and $\delta q_{n}^{\prime}(x)$ assuming that $K_{\text {eff }}^{2}$ is a small parameter.

${ }^{4}$ I. L. Drichko, A. M. D’yakonov, A. M. Kreshchuk, T. A. Polyanskaya, I. G. Savel'ev, I. Yu. Smirnov, and A. S. Suslov, Fiz. Tekh. Poluprovodn. 31, 451 (1997) [Sov. Phys. Semicond. 31, 451 (1997)].

${ }^{5}$ V. I. Talyanskii, J. M. Shilton, M. Pepper, C. G. Smith, C. J. B. Ford, E. H. Linfield, D. A. Ritchie, and G. A. C. Jones, Phys. Rev. B 56, 15180 (1997).

${ }^{6}$ G. R. Nash, S. J. Bending, Y. Kershaw, K. Eberl, P. Grambow, and K. von Klitzing, Surf. Sci. 361/362, 668 (1996). 
${ }^{7}$ J. M. Shilton, D. R. Mace, V. I. Talyanskii, M. Pepper, M. Y. Simmons, A. C. Churchill, and D. A. Ritchie, Phys. Rev. B 51, 14770 (1995).

${ }^{8}$ M. Rotter, A. V. Kalameitsev, A. O. Govorov, W. Ruile, and A. Wixforth, Phys. Rev. Lett. 82, 2171 (1999).

${ }^{9}$ M. Rotter, C. Rocke, S. Böhm, A. Lorke, A. Wixforth, W. Ruile, and L. Korte, Appl. Phys. Lett. 70, 2097 (1997).

${ }^{10}$ M. Rotter, A. Wixforth, W. Ruile, D. Bernklau, and H. Riechert, Appl. Phys. Lett. 73, 2128 (1998).

${ }^{11}$ M. Rotter, A. Wixforth, A. O. Govorov, W. Ruile, D. Bernklau, and H. Riechert, Appl. Phys. Lett. 75, 965 (1999).

${ }^{12}$ V. L. Gurevich and B. D. Laikhtman, Zh. Éksp. Teor. Fiz. 46, 598 (1964) [Sov. Phys. JETP 19, 407 (1964)]; V. L. Gurevich, Fiz. Tekh. Poluprovdn. 2, 1557 (1968) [Sov. Phys. Semicond. 2, 1299 (1969)].

${ }^{13}$ J. R. A. Beale, Phys. Rev. 135, 1761 (1964).

${ }^{14}$ P. K. Tien, Phys. Rev. 171, 970 (1968).

${ }^{15}$ P. N. Butcher and N. R. Ogg, J. Phys. D 2, 333 (1969).

${ }^{16}$ Yu. V. Gulyaev, Fiz. Tverd. Tela 12, 415 (1970) [Sov. Phys. Solid State 12, 328 (1970)].

${ }^{17}$ R. M. White and T. W. Voltmer, Appl. Phys. Lett. 8, 40 (1966).

${ }^{18}$ Yu. V. Gulyaev and V. V. Denisenko, Fiz. Tverd. Tela. 14, 1475 (1972) [Sov. Phys. Solid State 14, 1264 (1972)].

${ }^{19}$ R. C. Ho and C. L. Chen, J. Appl. Phys. 47, 3403 (1976); V. G. Mozhaev and I. Yu. Solodov, Akust. Zh. 26, 433 (1980) [Sov. Phys. Acoust. 26, 433 (1980)]; A. L. Belostotskii and L. A. Fedyukhin, ibid. 31, 433 (1985) [ibid. 31, 433 (1985)].
${ }^{20}$ K. A. Ingebrigtsen, J. Appl. Phys. 41, 454 (1970); A. L. Efros and Yu. M. Galperin, Phys. Rev. Lett. 64, 1959 (1990); V. I. Fal'ko, S. V. Meshkov, and S. V. Iordanskii, Phys. Rev. B 47, 9910 (1993); S. H. Simon, ibid. 54, 13878 (1996).

${ }^{21}$ A. V. Chaplik, Pis. Zh. Tekh. Fiz. 10, 1385 (1984) [Sov. Tech. Phys. Lett. 10, 584 (1984)]; L. I. Magarill and A. V. Chaplik, Zh. Éksp. Teor. Fiz. 93, 2257 (1987) [Sov. Phys. JETP 66, 1289 (1987)].

${ }^{22}$ B. A. Auld, Acoustic Fields and Waves in Solids (Wiley, New York, 1973).

${ }^{23}$ A. V. Chaplik, Zh. Éksp. Teor. Fiz. 62, 746 (1972) [Sov. Phys. JETP 35, 395 (1972)].

${ }^{24}$ This formula can be easily obtained in a linear theory by comparing a general expression for the SAW absorption $\langle j E\rangle$ and the formula (1); here $\langle\cdots\rangle$ means averaging over $\lambda$.

${ }^{25}$ G. Weinreich, Phys. Rev. 107, 317 (1957).

${ }^{26} \mathrm{~V}$. A. Vyun (unpublished).

${ }^{27}$ E. Yablonovich, D. M. Hwang, T. J. Gmitter, L. T. Florez, and J. P. Harbison, Appl. Phys. Lett. 56, 2419 (1990).

${ }^{28}$ M. K. Balakirev, in Nonlinear Phenomena in Solids-Modern Topics, edited by M. Borisov (World Scientific, Singapore, 1985), p. 191.

${ }^{29}$ M. K. Balakirev, S. V. Bogdanov, and L. A. Fedyukhin, Zh. Tekh. Fiz. 50, 2429 (1980).

${ }^{30}$ C. W. Skorupka, L. M. Pecora, T. L. Carroll, and T. M. Tritt, Phys. Rev. B 42, 9252 (1990); I. L'Heureux and F. Guillon, Phys. Rev. A 45, R5366 (1992). 